\title{
THE CANADIAN EXPERIENCE
}

\author{
CÉLINE MERCIER \\ McGill University, Québec
}

Céline Mercier has pursued studies in psychology, anthropology, and psycholinguistics. She received her $P h D$ in Psychology from the Université Louis Pasteur in Strasbourg. She is currently Associate Professor at the Department of Psychiatry, McGill University, Senior Researcher at the Psychosocial Research Division, Douglas Hospital Research Centre, and Senior Consultant at the Montreal World Health Organization Collaborating Centre. She chairs the Committee on Quality of Life for the World Association for Psychosocial Rehabilitation and is Vice-President of the Québec Mental Health Commintee. Dr. Mercier heads a research program on the organization and evaluation of mental health services. Her interests lie mainly in the development of evaluation models adapted to community approaches and in the use of quality of life indicators for evaluating mental health and rehabilitation services. She has evaluated a number of programs including prevention, detoxification, substance abuse rehabilitation, crisis teams, first-line teams, work rehabilitation and community support programs in mental health, and services to the homeless. Her current work concems the development of quality assurance programs; satisfaction studies with patients, family members, and mental health workers; and the validation for use in Canada of the Wisconsin Qualisy of Life Index, both English and French versions. Dr. Mercier has published many articles and has served as a national and intemational consultant. In 1990 she was awarded a Bursary of Excellence in Evaluation from the Québec Council for Social Research.

\section{INTRODUCTION}

The topic of my presentation is ambitious and very risky. My purpose is to identify in the specialized literature studies that have been conducted by Canadian researchers, with Canadian samples, and to consider them in the context of overall development in the field of quality of life measurement in individuals with severe mental illness. In this enterprise the risks are twofold: ignorance of important contributors and oversimplification. I may neglect to mention some people who are active in the field. I apologize in advance to those I fail to mention and I will be grateful to anyone who can help me rectify the situation. As for oversimplification, I am able to refer only briefly to my colleagues' works and it is quite possible that these quick references will be perceived as very rough statements. I will also limit myself to Canadians who have been involved in research in the mental health domain, even though many other Canadians are well known in such areas as quality of life and health prevention, measurement issues, utility approaches, research into cancer and into intellectual handicaps, and many more general issues.

The following overview of the Canadian experience and contributions to the research on quality of life in severe mental illness is based on a previous literature review which I worked on in order to familiarize francophone audiences with the 
field (Mercier, 1994b; Mercier \& Corten, 1994). In this review, published studies were divided between theories and models, descriptive and comparative studies, evaluative studies, and measurement issues. I will follow this plan, putting emphasis on the Canadian contributions and the current evolution in the field.

\section{LITERATURE REVIEW}

\section{The First Steps}

To my knowledge, the first paper referring to quality of life in the context of severe mental illness was published by Bigelow and Beiser (1978). It appeared a couple of years before the now classic study by Stein and Test (1980) on the impact of the program "Training in Community Living" on the life circumstances and satisfaction with life of deinstitutionalized psychiatric patients. In their evaluation of the Greater Vancouver Mental Health Service (GVMHS), Bigelow and Beiser tested the hypothesis "that GVMHS improved the quality of life for high risk patients" (p. 10). The authors conducted "unstructured interviews with current patients in order to discover what the key dimensions of quality of life are, in their own terms" (p. 11). In contrast with their hospital experiences, the patients appreciated having more control over their time and destiny and had more contact with family and friends. They made positive comments regarding the staff visits to their homes and their welcoming attitude. Finally, they emphasized their "need for a place in which they could feel welcome" (p, 11) and for purposeful activity.

\section{Models and Theories}

Bigelow, Brodsky, Steward, and Olson (1982) were also among the first to propose quality of life as a dependent variable in the evaluation of mental health services. They developed a theoretical basis in relation to quality of life assessments so that results from outcome studies could be translated by mental health staff in terms of psychosocial strategies and interventions. According to Bigelow's team model (Bigelow et al., 1982), quality of life consists of fulfilling individual needs and meeting social demands. Quality of life is the result of both the satisfaction of personal needs, using the resources available in the environment, and the individual's performance, or the realization of one's abilities in response to society's demands. Two other Canadians have also worked on models for identifying significant components of subjective quality of life. The model proposed by Corten, Mercier, and Pelc (1994) is derived from empirical data. Using a principal component analysis, we identified three dimensions as the major determinants of subjective quality of life: hedonism, achievement, and conformity. Awad (1992) has developed a conceptual model for measuring the quality of life of medicated patients with schizophrenia. According to this model, the major determinants of quality of life are schizophrenic symptoms and their severity, adverse effects of antipsychotic medications, and psychosocial performance. This model was validated in a study with 62 stable schizophrenic outpatients maintained on antipsychotic medication (Awad, Voruganti, \& Heselgrave, 1997):

Preliminary results based on multiple regression using subjective quality of life as an outcome variable indicated that the severity of schizophrenic symptoms (partial $r^{2}=0.32, \quad p<.0001$ ), akathisia-related subjective distress (partial 
$r^{2}=0.11, p<.001$ ), and neuroleptic dysphoria (partial $r^{2}=0.06, p<.05$ )

accounted for nearly half of the variance (p. 41).

These three models are quite different from those proposed and studied by Franklin, Simmons, Solovitz, Clemons, and Miller (1986) or by Lehman (1983a). According to Lehman's model, an overall feeling of well-being is associated primarily with satisfaction with life circumstances, and secondly with objective living conditions and sociodemographic characteristics. Franklin's model refers to variables related to adaptation. These wide differences in conceptualization of quality of life are quite representative of the lack of agreement on the definition of quality of life and the lack of adequate conceptual models (Awad et al., 1997). Surprisingly, however, there is much convergence regarding the empirical data.

\section{Descriptive Studies}

Results from the Canadian surveys tend to confirm what has been observed in the United States (Lehman, Reed, \& Possidente, 1982) regarding the factors that contribute to subjective quality of life. In the study done by Kearns, Taylor, and Dear (1987) in Hamilton, seven variables were related to greater satisfaction with life: being older, not living in a lodging home, having a certain degree of residential stability, being able to identify several significant relationships, not being on welfare, and having sufficient leisure activities. Coping was significantly correlated with satisfaction with life (self-assessed, $r=0.55$; assessed by caregiver, $r=0.41$ ). Conversely, Levitt, Hogan, and Bucosky (1990) identified the frequency of family contacts, satisfaction with social life, mental health, level of education, and number of hospital admissions in the previous year as the best predictors of quality of life. In a causal modelling analysis in which Lisrel was used with data collected in Montreal, the latent variable "Autonomy," defined as not living in a group home, higher level of global functioning, having enough money, and having at least one leisure activity, showed the strongest relationship with satisfaction with life as a whole (Mercier \& King, 1994). However, these objective indicators had much less influence on the assessment of quality of life than subjective ones: satisfaction with health, occupation, social relationships, and finances. These results compare well with American studies. Greater overall satisfaction with life is associated with greater satisfaction with health, daily activity and leisure, social relations, and financial situation. Objective indicators included age, financial situation, employment status, health condition, and frequency of interpersonal relationships.

\section{Comparative Studies}

Quality of life has been compared among subgroups of severely mentally ill people and between severely mentally ill persons and the general population. Studies comparing different subgroups of people with severe mental illness according to their environmental and living conditions have been carried out in Canada, the United Kingdom, and the United States, with similar conclusions. Individuals living in more restrictive settings have more negative perceptions of their quality of life compared to those living in less restrictive environments (Lehman, Possidente, \& Hawker, 1986; 1991; Levitt et al., 1990; Mercier, 1989; Simpson, Hyde, \& Faragher, 1989; Warner \& Huxley, 1993a). The same convergence of results is observed regarding geographic location. People with severe 
mental disorders living in rural areas in Mississippi (Sullivan, Wells, \& Leake, 1991). New York State (Baker \& Intagliata, 1982), or Abitibi-Temiscamingue (Mercier, Tempier, \& Renaud, 1992) express higher levels of satisfaction with life in general than those living in urban areas.

Canadian studies have compared mean quality of life ratings from samples of severely mentally ill people with the general population and with subsamples (Aubry \& Myner, 1996; Mercier et al., 1992; Tempier, Caron, Mercier, \& Leouffre, in press). As elsewhere, the evaluation of life-as-a-whole is lower among clinical populations than among the general population. The results of a study of persons with psychiatric disabilities in housing programs in Ottawa-Carleton showed persons with psychiatric disabilities reporting lower general life satisfaction than other community residents (Aubry \& Myner, 1996). The same conclusion can be drawn from a study comparing samples of severely mentally ill people living in Montreal, in a suburb, and in a remote area of Quebec. All three groups expressed less satisfaction with life in general than the general population. However, all three groups also had a slightly more positive perception of their quality of life in all domains pertaining to housing (the place they lived; neighbourhood, services, and facilities in the area).

Some divergences are observed between American and Canadian surveys when we consider the situation of severely mentally ill people compared to other less disadvantaged groups (Lehman, Reed, \& Possidente, 1982). In the United States the ratings of severely mentally ill persons are the lowest of all the deprived groups, On a 7-point Likert scale the general American population rated their life as a whole at 5.5 , while people with low socioeconomic status rated it at 5.1 , single parents at 5.0, and Afro-Americans at 4.8 (Andrews \& Withey, 1976). The ratings of the clinical population through different urban samples ranged between 4.4 and 4.9. A Quebec study (Tempier et al., in press) which compared the subjective quality of life of severely mentally ill individuals receiving community support services ( $N=60)$ with both a random sample from the general population $(N=263)$ and with welfare recipients $(N=79)$, found ratings for life-as-a-whole were lower for the severely mentally ill $(5.2)$ compared to the general population $(5.5)$, but were higher than ratings for welfare recipients $(5.0)(p<.05)$. Ratings from the clinical population were higher than those of welfare recipients in the domain of residence $(p<.01)$. financial situation $(p<.01)$, and daily activities $(p<.01)$. This may be explained by the fact that individuals suffering from mental illness may have lower aspirations. Moreover, because they benefit from community services, they may be in a better financial situation than their welfare counterparts and participate in various programs that provide daily activities and opportunities for social contacts.

In the field of descriptive studies, Health Canada (1996b) compared patients with different psychiatric disorders and patients with chronic physical and mental illnesses. On a subjective measure of quality of life, "significant differences were found among ratings of the patients with schizophrenia, bipolar disorder, and depression, the subjects with depression and bipolar disorder being more likely to report lower [quality of life]" (p. 101). No significant differences were found between patients with schizophrenia and a comparison group of haemodialysis patients. However, the opposite trend occurred when groups were compared on 
objective measures. Schizophrenic patients experienced more aversive life circumstances than either of the affectively disturbed groups. This study raises the crucial issue of the role of symptoms in the assessment of quality of life and the value of self-reports. Papers from many countries have been published on these issues without clear conclusions (Awad, 1992; Browne et al., 1996; Halford, Schweitzer, \& Varghese, 1991; Heslegrave, Voruganti, \& Awad, 1995; Heselgrave, Awad, \& Voruganti, 1997; Lehman, 1983b; Mechanic, McAlpine, Rosenfield, \& Davis, 1994; Rosenblat \& Attkisson, 1993; Sainfort, Becker, \& Diamond, 1996; Sullivan et al., 1992; Warner \& Huxley, 1993b).

\section{Evaluation Research}

Canadian researchers have examined the impact of services on objective and subjective quality of life. Goering. Wasylenki, Farkas, Lance, and Ballantyne (1988), evaluated the impact of a rehabilitation-oriented case management program on the objective quality of life of patients 6 months and 2 years after they entered the program. At the 6-month follow-up, patients enrolled in the program showed better occupational functioning than the control group. At the 2-year follow-up. patients in the program were more likely than the control patients to have better occupational functioning, to live more independently, and to be less socially isolated. Pinkney, Gerber and Lafave (1991) reported that $96 \%$ of those discharged from two hospital-based rehabilitation programs considered their objective quality of life to have improved since they left the hospital. Mercier et al. (1992) observed the impact of community support services on the quality of life of long-term psychiatric patients living in a remote area. The subjective perception of quality of life of these patients was comparable with a matched group living in a neighbouring city with only out-patient services. However, the objective living conditions were much less favourable for the group participating in the community support program.

\section{Specific Contributions}

Transcultural studies. I know of three research programs that have attempted to compare quality of life between different countries and cultures. One comparison has been made between England and the United States (Warner \& Huxley, 1993a) and two others between Canada and (a) Cuba, Mexico, and the United States (Vandiver, in press), and (b) Belgium and France (Mercier \& Corten, 1989). Cross-cultural studies are difficult to conduct given the number of factors that have to be considered. However, they have great potential for studying the impact of different mental health systems on the general condition of individuals with mental health problems (Beiser, Shore, Peters, \& Talum, 1985).

Scale development, translation, and cultural validation. Specific scales have been developed by Bigelow and colleagues (Bigelow, Gareau, \& Young, 1990; Bigelow, McFarland, \& Olson, 1991; see also Health Canada, 1996b). Roy Brown (1988) has developed an extended interview protocol for studying quality of life for people with learning difficulties, David Evans and his colleagues are the authors of the Quality of Life Questionnaire (Evans, Burns, Robinson, \& Garell, 1983). 
Well known scales that have been translated from English and validated include Lehman's Quality of Life Interview (Ely, 1991), Baker and Intagliata's Satisfaction with Life Domains Scale (Caron, Mercier, \& Tempier, 1997), the Wisconsin Quality of Life Index (Diaz \& Mercier, 1996), the Quality of Life in Depression Scale (Mercier, Piat, \& Pecknold, 1996), and Diener's Satisfaction with Life Scale (Blais, Vallerand, Pelletier, \& Brière, 1989).

\section{FUTURE DIRECTIONS}

Canadian research in the field of quality of life is representative of what is going on elsewhere. However, it seems we are now moving to a second generation of studies characterized by the convergence of health-related quality of life and overall quality of life perspectives. In the 1980 s the first studies on quality of life in mental health developed on a different basis than did studies of physical health. At the beginning the studies on quality of life were prompted by the deinstitutionalization movement and concerns for the life circumstances of expatients. The first generation of scales therefore focused strongly on descriptive and comparative studies of objective life circumstances, responses to needs, and satisfaction with different domains of life. Quality of life was used as an outcome indicator for new services in the community for severely mentally ill people. Efforts in psychosocial rehabilitation also supported the inclusion of functional measures in the assessment of quality of life.

Today transformations in the larger health system (including budgetary cuts, reductions in beds, and the shift to ambulatory care), as well as in sectors with restricted accessibility (such as housing and welfare), are more at issue. Quality of life is seen in the context of policies and new practices and their impact on health conditions, well-being, and socioeconomic conditions. Moreover, there is increasing need and demand for evaluation of service efficiency and economic studies about alternative models of service delivery. This calls for more operational measurement instruments, such as those used in the health-related quality of life field.

Another factor which plays a role in the extension of the quality of life concept is the current interest in quality of life as an indicator in clinical trials. The fact that quality of life can now be used as an argument for marketing new neuroleptics could have tremendous impact. On the one hand, psychopharmacology is concerned with the symptomatic dimension of the illness which relates more to health-related quality of life. On the other hand, it is because new neuroleptics are hoped to have an impact on negative symptoms, general functioning, and ultimately, on social integration, that their evaluation according to their impact on quality of life is relevant. This new context is a factor promoting the integration of the health-related quality of life approach in the assessment of quality of life of individuals with schizophrenia. As stated by Awad et al. (1997):

The recent pressures on the pharmaceutical industry to provide quality-of-life and pharmacoeconomic data about new drugs are creating major interests in the concepts as well as the methodology (p. 45).

If we are moving from a definition of quality of life in terms of satisfaction with life circumstances and fulfilment of needs-well suited for planning and organization of support services in the community-to a multidimensional one, as 
in health-related quality of life, we are in a good position to discuss some basic issues:

- To what extent are the proposed dimensions of physical and occupational function, psychological state, social ability, somatic comfort (Schipper \& Levitt, 1985), disease symptoms and treatment side effects, functional status, psychological distress, social interaction, sexuality and body image, and satisfaction with medical treatment (Aaronson, 1986) relevant for individuals with schizophrenia?

- Do we need, or do we already have, a multidimensional, disease-specific instrument? Are we interested in a generic scale?

- Researchers now increasingly work in networks concerning specific pathologies or specific scales. Do we want to create or join such a network?

-What is needed for the future development of the field? Do we need standards from the general population with comparable socioeconomic status groups, or comparisons with other clinical groups? 